\title{
EVOLUTION OF SENSORY ANTENNAL STRUCTURES IN THE ENSIGN SCALE INSECTS (HEMIPTERA COCCOMORPHA ORTHEZIIDAE) $\left(^{1}\right)$
}

\author{
(*) İmamoğlu Vocational School, Çukurova University, Adana, Turkey \\ (*) Plant Protection Institute, Centre for Agricultural Research, Hungarian Academy of Sciences, Budapest, Hungary \\ (***) Laboratory of Applied Entomology, Graduate School of Bioagricultural Sciences, Nagoya University, Japan \\ Corresponding author: e-mail: szita.eva@agrar.mta.hu
}

Kaydan M.B., Vea I.M., Szita É. - Evolution of sensory antennal structures in the ensign scale insects (Hemiptera Coccomorpha Ortheziidae).

The Ortheziidae (Hemiptera: Sternorrhyncha: Coccomorpha) are considered one of the most ancient families of Coccoidea. In this study, the antennal structures of species belonging to the Ortheziidae are examined and combined with data from a previous phylogenetic study as well as habitat preference data. Illustrations of the antennal structures (with a focus on the distribution of setae and specialized sensilla) for the type species of all genera in the Ortheziidae are provided. The three main ortheziid lineages can be recognized by their sensilla: (i) the Ortheziinae all possess coeloconic sensilla; (ii) the Newsteadiinae, which only includes Newsteadia, is characterized by a different type of coeloconic sensilla, here named Newsteadia-type coeloconic sensilla, and (iii) a clade that includes the Mixortheziinii, Nipponortheziini and Ortheziolini, all possess only basiconic sensilla.

KEY WORDS: thermoreceptor, hygroreceptor, leaf litter, hypogeal insect, habitat.

\section{INTRODUCTION}

The Ortheziidae, or ensign scale insects, are considered one of the most ancient families of Coccoidea (KoTEJA, 1986; Vea \& Grimaldi, 2012). Females are distinctive, possessing well-developed legs and antennae, and have much of the body cloaked in bundles of extravagant, white wax secretions, giving them a peculiarly ornate appearance. There are about 208 described species of Ortheziidae to date, classified within 21 genera (including four extinct genera) (VEA \& GRIMALDI, 2012). Although a few species of ortheziids are serious plant pests, such as the greenhouse ensign scale (Insignorthezia insignis (Browne)), most occur sporadically in leaf litter (presumably feeding on roots and fungal mycelia), and some are assumed to feed on mosses and lichens, habitats that are putatively the most primitive in Coccoidea (Koteja, 1986; KozÁr, 2004; VeA \& GRIMALDI, 2012).

Koteja (1985), based on an intuitive analysis of morphological variation and habitat of recent scale insects, hypothesized that the evolution of Coccoidea occurred in two main ecological stages. The first occurred after the split from the "homopteran" stock (probably during the Carboniferous), scale insects first lived in the forest litter on a "mixed" diet, sucking out liquid from the surface and from living and decaying plant tissues. During this period of soil habitat, the leg became a digging organ (one claw, one-segmented tarsus, functional tibio-tarsal joint), females lost their wings and became paedomorphic and with reduced life stages; males became dipterous and poly-

\footnotetext{
${ }^{1}$ Original scientific contribution presented and discussed at XIV International Symposium on Scale Insect Studies, Catania-Italy, 13-16 June 2016.
}

morphic without functional mouthparts, and acquired a different life cycle (quiescent stages reminiscent of complete metamorphosis) that shortened that developmental time. These transformations probably led to a primary radiation creating numerous new families during the Permian or Triassic. The second stage of evolution started during the early evolution of flowering plants (Jurassic) and continued to the present and the evolutionary trends radically changed: coccoids became true plant parasites. Most of the groups started to climb on plants and each of them settled and integrated their own endosymbionts. The switch from soil litter to plant habitat took place during a long period of time and independently within various groups (KOTEJA, 1985); as a result, recent scale insects are extremely differentiated and some groups still continue the primary mode of life (hypogeal insects such as some Margarodidae sensu stricto, Rhizoecidae and some members of Ortheziidae).

Insect antennae possess various types of specialized sensilla that can function as chemoreceptors, thermoreceptors and/or hygroreceptors (KEIL, 1999) and play an important role in orientation (SCHNEIDER, 1964). Their number and distribution on antennal segments are potentially significant taxonomically (LARINK, 1986) and phylogenetically (WALTHER, 1983). Two morphological types, namely basiconic sensilla (bs) and coeloconic sensilla (cs), are present in many insect groups and may be responsible for humidity and thermo-detection (SCHNEIDER, 1964). Basiconic sensilla (sensory pegs or cones) are trichoid sensilla without any specialized basal membrane. Coeloconic sensilla (sensory pit-peg) are thin walled cones on the floor of depressions in the antennal cuticle. In crosssection this structure appears as double-walled, with pores and peg attached to several neurons. Despite their obvious structural differences, KоTEJA (1980) suggested a homology 
between $b s$ and $c s$, with $b s$ probably being the primary type because they occur mostly in archaeococcoid families, and cs being a secondary transformation of the basiconic-type and occurring generally in neococcoids (KOTEJA, 1980). Nevertheless, cs are found within the archaeococcoid family Ortheziidae, more specifically in the genera Orthezia, Arctorthezia and Newsteadia, while bs have been identified in Ortheziola (KоTEJA, 1980).

The non-overlapping presence of the two main types of antennal sensilla and the variety of habitats within the Ortheziidae provide a good model to examine the evolution of these sensilla in relation to habitat. As such, this study aims to determine: (i) whether distribution of $b s$ and $c s$ across ortheziid genera, (ii) whether there is any association between the types of sensilla and the currently recognized generic concepts, (iii) whether these antennal characters affect the phylogenetic hypotheses for the major ortheziid clades proposed by VEA \& GRIMALDI (2012), and (iv) whether there is an association between the sensilla and habitat of ortheziid species. We also provide illustrations of the antennal structures for the type-species of all genera within the family Ortheziidae, showing the distribution of setae and specialized sensilla on all the antennal segments.

\section{MATERIAL AND METHODS}

TAXA AND SPECIMENS EXAMINED

Here we provide illustrations of the antennae of the type species of each of the known genera of the family Ortheziidae. Several species were studied for the Newsteadiinae, even though it contains just one genus, because of variation in antennal segment number among species.
All examined specimens mounted on microscope slides that are deposited at the Hungarian Natural History Museum (HNHM), Budapest, Hungary, and the Plant Protection Institute (PPI), Centre for Agricultural Research, Hungarian Academy of Sciences, Budapest, Hungary. These collections were acquired mostly by the late Ferenc Kozár

\section{Phylogenetic ANALYsis}

\section{Taxon sampling and morphological characters}

The morphological matrix of VeA \& GRIMALDI (2012) was used as the basis of our analysis. In VEA \& GRIMALDI (2012), the character about the microsetae was defined and coded based on KozÁr (2004) definition. However, this character refers to the $b s$ of KotejA (1980). We therefore removed microsetal as a character from the original matrix and added the following new character:

Type of antennal sensilla. According to Koteja (1980), bs and $c s$ are two types of the same sensilla, being located as they occur on the same parts of the antennae and their occurrence does not overlap across species. States: $0=$ thinwalled cs (Fig. I., 1, 2); 1= thick-walled cs (Newsteadia type) (Fig. I., 3); $2=b s$ (Fig. I., 4, 5).

\section{Phylogenetic and ancestral reconstruction methods}

The final morphological matrix of 47 taxa and 70 characters was analyzed using Maximum Parsimony (MP) as the optimality criterion, using TNT (GolobofF et al., 2008) (command lines: hold 10000; mult $=$ tbr replic 10000 hold 100), with all characters treated as unordered and Matsucoccus set as the outgroup. Bootstrap supports were calculated with 10000 replicates (command line: resample boot replic 10000).

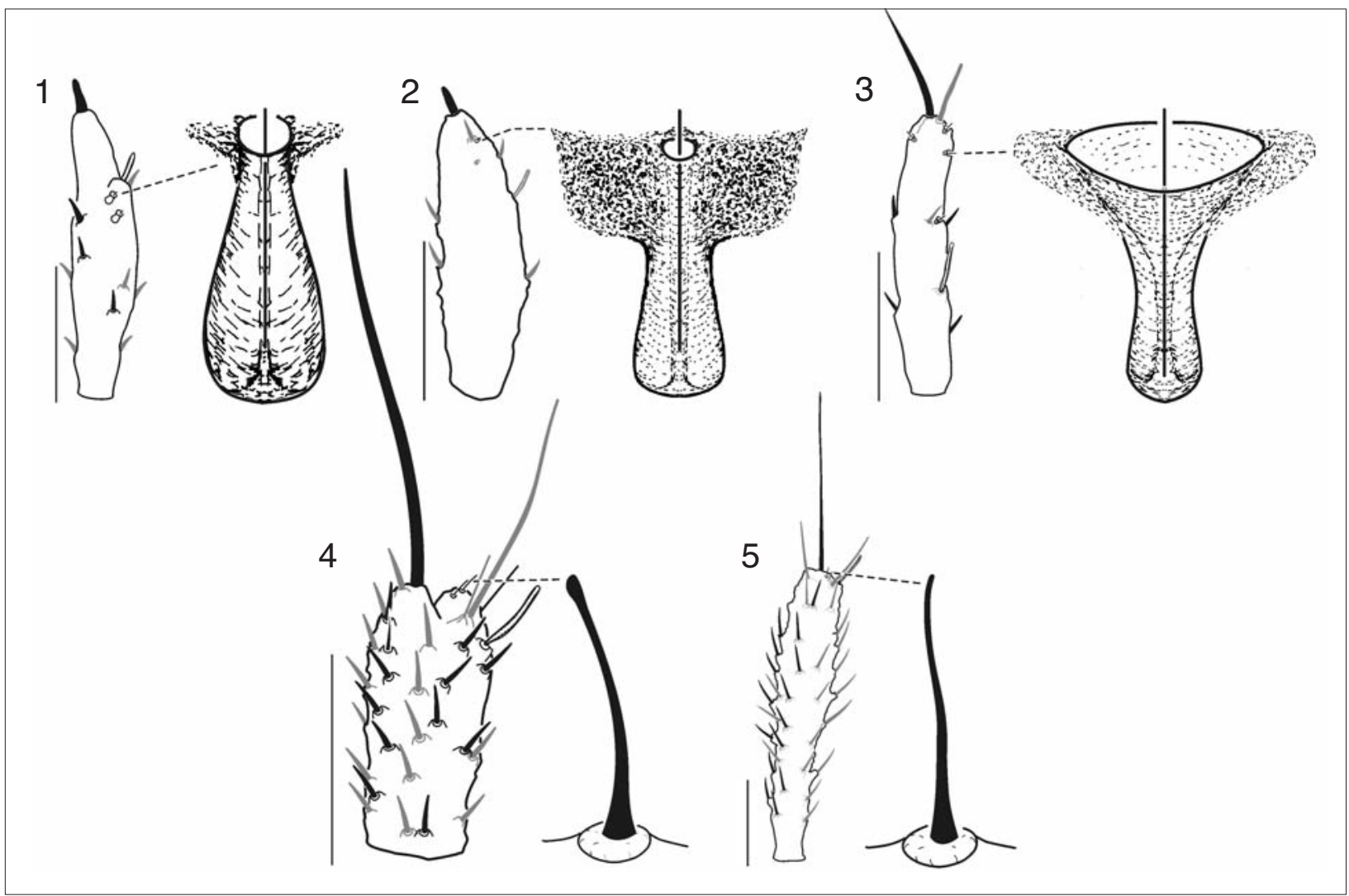

Fig. I - Sensilla types, 1, 2 -Coeloconic sensilla, 3 - Newsteadia-type coeloconic sensilla, 4 -Basiconic sensilla with capitate apex, 5 Basiconic sensilla with a blunt apex. Black setae on ventral side, grey setae on dorsal side. Bar $=100 \mu \mathrm{m}$. 
To examine any relationship between the type of antennal sensilla and habitat within the family, an ancestral reconstruction of character 70 was performed using the parsimony criterion in Mesquite version 3.01 (MADDISON \& MADDISON, 2014) using a separate matrix (Matrix 2) from which habitat information based on KozÁr (2004) was also coded as follows:

Aerial plant parts (0): species found feeding on stems and plant leaves.

Roots or ground dwelling (1): species found feeding on plant roots, or in leaf litter or on lichen (Character 70).

The new morphological matrix is available in Morphobank (O'LEARY \& KAUfMan, 2012) under Project 2181 (reviewer login at www.morphobank.com, login: 2181, password: Ortheziidae2015).

\section{RESULTS}

\section{ORTHEZIIDAE}

Description. Antennae 3 to 8 segmented; 7 or 8 segments in Ortheziinae, 3 in Ortheziolinae and Nipponortheziini, 4 in Mixortheziini and 3-7 in Newsteadiinae. Antennal bases separated from eyes except for Ortheziolinae. Setae spinelike, hair-like or clavate. Apical and subapical setae strongly blunt or hair-like. Sensory pore (campaniform sensillum) dorsally on second segment (pedicel). Sensory fleshy setae on apical segment(s). Both $b s$ and $c s$ generally present on apical segment and pedicel ventrally.

\section{ORTHEZIINAE}

Description. Antennae 7 or 8 segmented. Setae spine like or hair-like. Apical seta short, strong, blunt; subapical setae hair-like. Sensory fleshy setae present on apical segments. Cs may be present on any segment ventrally. Cs thin, with hardly visible seta, inserted at bottom of invagination with narrow opening. Bs absent.

\section{ARCTORTHEZIINI}

Arctorthezia occidentalis (Douglas) (Fig. II, 1)

Material examined - 1 \&, USA, New Mexico, Magdalena Mts., ex. Compositae, 15.ix.1994, leg. F. Kozár [4324 PPI]; 4 우, Canada, Ferry Creek, ex. moss, 7.vii.1988, leg. F. Kozár [3309 PPI].

Description - Antennae 7 or 8 (rarely 6) segmented; length of antennal segments: I 189-257, II 144-227, III 102-134, IV 64-123, V 61-113, VI 67, VII 70-102, and apical 176-250 $\mu \mathrm{m}$ long; all segments covered with 2-4 very robust, spine-like setae, longest spine 11-16 $\mu \mathrm{m}$

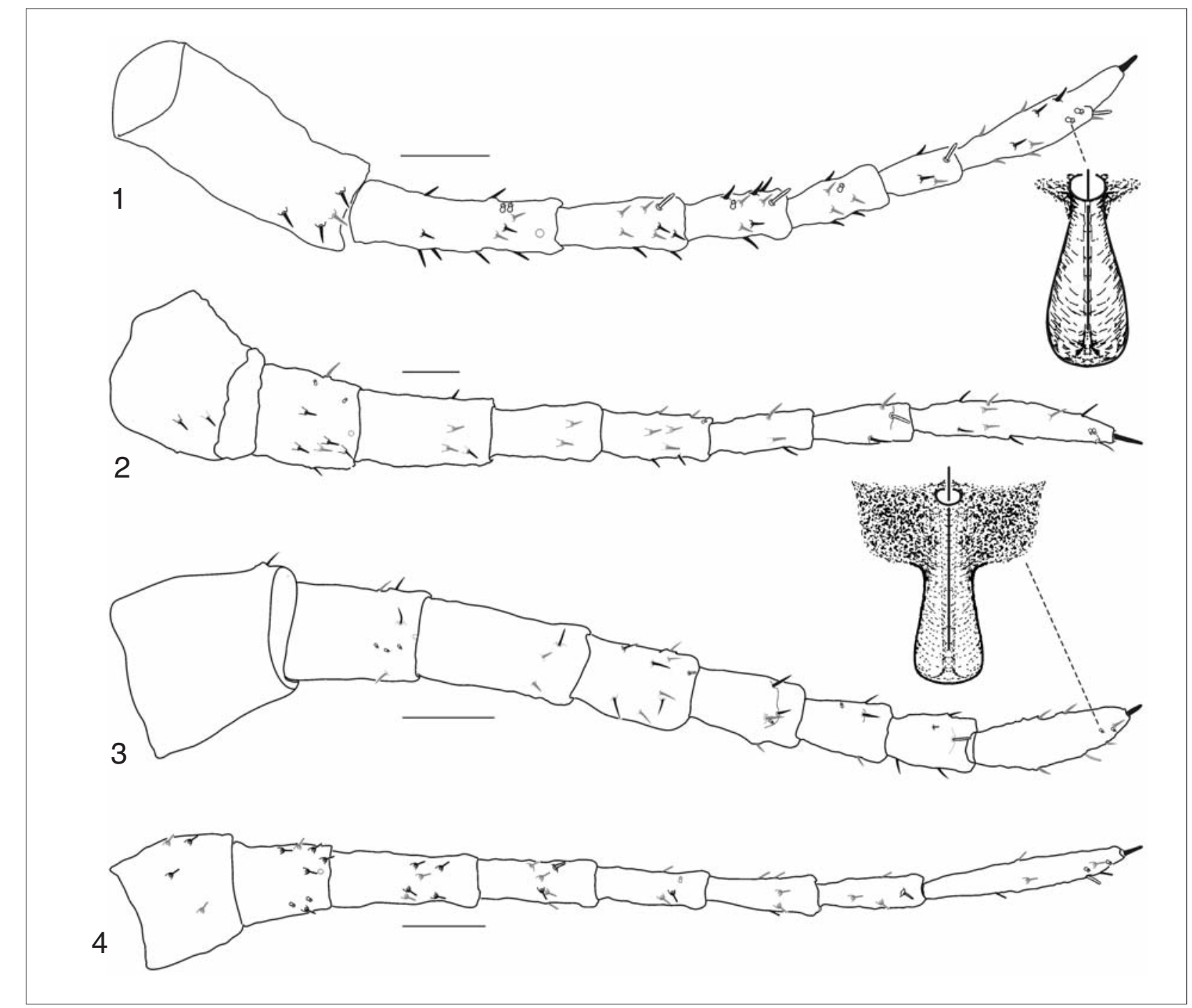

Fig. II - Coeloconic sensilla found in Ortheziinae. 1 - Arctorthezia occidentalis (Douglas), 2 -Insignorthezia insignis (Browne), 3 - Orthezia urticae (Linneaus), 4 - Praelongorthezia praelonga (Douglas). Black setae on ventral side, grey setae on dorsal side. Bar $=100 \mu \mathrm{m}$. 
long; apical spine of antennae thick and blunted, 11-20 $\mu \mathrm{m}$ long; subapical seta absent; sensory fleshy seta near apical seta and on preapical segment, 13-20 $\mu \mathrm{m}$ long each; 1 cs on segments III, IV, V, VI and 3 on apical segment. Bs absent.

\section{ORTHEZIINI}

\section{Insignorthezia insignis (Browne) (Fig. II, 2)}

Material examined - 1 , South Africa, Nelspruit, Nat. Reserve, 25.x.1986, leg. Endrődy [4882 PPI]; 1 , Tanzania, USA River, 1-25.iv.1965, leg. Szunyoghy [99 PPI]; 3 ․ Tanzania, leg. unknown [634 PPI]; 1 옹 Tanzania, 4.ii.1985, leg. Peregovits [590 PPI]; 3 qo : USA, U.S. Dept. Agr., greenhouses, on Coleus sp., 21.i.1919, leg. N. Perrine; 1 O: Kenya, 26.iii.1978 [471 PPI].

Description - Antennae 7 or 8 (rarely 6) segmented; length of antennal segments: I 112-123, II 87-93, III $107-$ 118, IV 97-103, V 102-115, VI 82-98, VII 77-87, and apical 154-180 $\mu \mathrm{m}$ long; all segments covered with 2-6 spine-like, straight setae, longest seta 6-10 $\mu \mathrm{m}$ long; apical seta 24-30 $\mu \mathrm{m}$ long; subapical seta absent; sensory fleshy seta on apical segment and on preapical segment, each 16-19 $\mu \mathrm{m}$ long; 1 or 2 cs on antennal segment II, 1 on V, 2 on apical segment. Bs absent.

\section{Orthezia urticae (Linneaus) (Fig. II, 3)}

Material examined - 1 , , Germany, Thuringia, Jena Distr., Pine forest near Zöllnitz, ex. Melampyrum pratense, 29.vii.1989, leg. G. Köhler; 2 qq Hungary, Csobánctető, ex. Umbelliferae, 22.viii.2002, leg. Kozár [6922 PPI]; 1 , Hungary, leg. Kozár [4982 PPI]; 1 9, Hungary, Nagykovácsi, Nagyszénás Mt., ex. Thesium sp., 9.viii.2004, leg. B. Nagy [7068 PPI]; 1 q, Hungary, Szin, ex. Dorycnium germanicum, 30.vii.1987, leg. Kozár [3097 PPI]; 1 ㅇ, Switzerland, Romoos, Wiese, 13.v.1994, leg. Rézbányai [6147 PPI].

Description - Antennae 8 (rarely 6 or 7) segmented; length of antennal segments: I 160-175, II 144-185, III 188237, IV 140-192, V 128-170, VI 108-144, VII 103-144, apical 185-237 $\mu \mathrm{m}$ long; all segments covered with 3-6 spine-like, straight setae; apical seta a strong blunt spine, 12-15 $\mu \mathrm{m}$ long; subapical seta absent; sensory fleshy seta present on apical and subapical segments, each 13-19 $\mu \mathrm{m}$ long, plus a smaller seta on segment IV; 2 or $3 c s$ on segment II, 1 on V and V, 2 on apical segment. Bs absent.

\section{Praelongorthezia praelonga (Douglas) (Fig. II, 4)}

Material examined - 3 오, Congo, Brazzaville, ex. Citrus sp., 2.v.2008, leg. A. Kiyindou [8195 PPI].

Description - Antennae 8 (rarely 6 or 7 ) segmented; length of antennal segments: I 102-123, II 87-103, III 107175, IV 92-129, V 102-123, VI 102-123, VII 97-113, and apical segment 206-237 $\mu \mathrm{m}$ long, all segments covered with 4-10 spine-like, straight setae, longest seta 7-12 $\mu \mathrm{m}$ long; apical seta short, blunt, 19-25 $\mu \mathrm{m}$ long; subapical seta absent; sensory fleshy seta present on apical and subapical segments and on segment IV, each 22-24 $\mu \mathrm{m}$ long; also 2 cs present on segment II, 1 on V, 2 on apical segment. $B s$ absent.

\section{NeWSTEAdinAe}

NEWSTEADIINI

Description - Antennae 3 to 8 segmented. Setae on antennae spine-like or hair-like. Apical and subapical setae long hair-like. One sensory fleshy seta present on apical segment. Cs structurally different from Ortheziinae: thickwalled, named here Newsteadia-type cs. Each sensilla with a thin seta, inserted at bottom of invaginations with wide thick-walled cone-shaped opening (Figures I, 3, III, 3), located on ventral side of antennae, with 2-4 on apical segment and on 1 or 2 on segment II, occasionally on segment III. Bs absent.

Newsteadia africana Miller \& Kozár (Fig. III, 1)

Material examined - 1 \&, Holotype, South Africa, Nelspruit Nature Reserve, litter, 18.xii.1986, leg. Y.S. Endrődy [4898 PPI]; 1 \%, Paratype: South Africa, Kitsuk Forest St., 26.x.1968, leg. Y.S. Endrödy [4895 PPI]; 3 우, Paratypes: South Africa, Dweza Forest, 27.ii.1986, leg. Y.S. Endrődy [4897 PPI]; 1 q, Paratype: South Africa, Dweza Forest, 6.xii.1978, leg. Y.S. Endrődy [4833 PPI].

Description - Antennae 7 segmented; length of antennal segments: I 145-205, II 155-168, III 31-41, IV 31-41, V 36 , VI 41, and apical 175-242 $\mu \mathrm{m}$ long; with 6-8 hair-like setae on segment I; 4 hair-like setae on segment II; without setae on segment III, IV, V, and VI; 6 hair-like setae present on apical segment, longest seta $24-31 \mu \mathrm{m}$ long; apical seta hairlike, 100-118 $\mu \mathrm{m}$ long, subapical seta hair-like, 55-58 $\mu \mathrm{m}$ long; with 1 sensory fleshy seta on apical segment, 26-38 $\mu \mathrm{m}$ long; 1 Newsteadia-type cs present on segment II, 3 on apical segment. $B s$ absent.

\section{Newsteadia caledoniensis Kozár \& Konczné Benedicty} (Fig. III, 3)

Material examined - 1 ㅇ Holotype, 3 우 Paratypes: New Caledonia, Ile de Pius, litter, 18.v.1986, leg. J. Balogh [B.12 HNHM].

Description - Antennae 4 segmented; length of antennal segments: I 93-108, II 67-82, III 82-98, and apical 180-191 $\mu \mathrm{m}$ long, with 4-8 spine-like setae on all segments, longest setae $6 \mu \mathrm{m}$ long; apical seta thick and blunt, 70-74 $\mu \mathrm{m}$ long, subapical seta absent, sensory fleshy seta on apical segment, $8 \mu \mathrm{m}$ long; with 2 Newsteadia-type cs present on apical segment. $B s$ absent.

\section{Newsteadia floccosa (De Geer) (Fig. III, 3)}

Material examined - 1 9, Armenia, Dilizhan, 1600, 28.ix.1982, leg. O. Merkl [As 566 HNHM]; 1 \%, Finland, Helsinki, ex. moss, 5.v.2005, leg. Pócs [E-1555 HNHM, 11039 PPI]; 1 \&, Hungary, Esztergom, Strázsa Mt., D-vac, 23.ix.2011, leg. É. Szita [10088 PPI]; 1 q, Hungary, Lébény, soil sample from horn beam (Carpinus) forest, 0205.ix.1997, leg. E. Horváth [6265 PPI]; 1 q, Hungary, Körös-Maros National Park, Bányarét, ex. moss, 1718.viii.1999, leg. Kozár [6020 PPI]; 1 q, Hungary, Nagykovácsi, Júlia-major, rocky grassland, 9-11.ii.2000, leg. F. Samu [6069 PPI]; 1 , Romania, Oltenia, litter, 25.x5.xi.2007, leg. J. Kontschán [10821/a PPI]; 1 †, Romania, Maramures Mts., 24.v.2007, leg. J. Kontschán [10922 PPI]; 1 ㅇ, Switzerland, Valais, s/Venayaz, $700 \mathrm{~m}$ a.s.1, 7.x.1980, leg. C. Besuchet [8864 PPI].

Description - Antennae 6 or 7 segmented, length of antennal segments: I 227-237, II 154-165, III 62-37, IV 4151, V 46-51, VI 46-51, and apical 186-216 $\mu \mathrm{m}$ long; with 28 spine-like setae present on all segments, longest seta $12-13 \mu \mathrm{m}$ long; apical seta $80-90 \mu \mathrm{m}$ long; subapical seta 41-47 $\mu \mathrm{m}$ long; sensory fleshy seta present on apical segment, each 36-41 $\mu \mathrm{m}$ long; with 2 Newsteadia-type cs present on segment II, 1 on III, 4 on apical segment. $B S$ absent. 


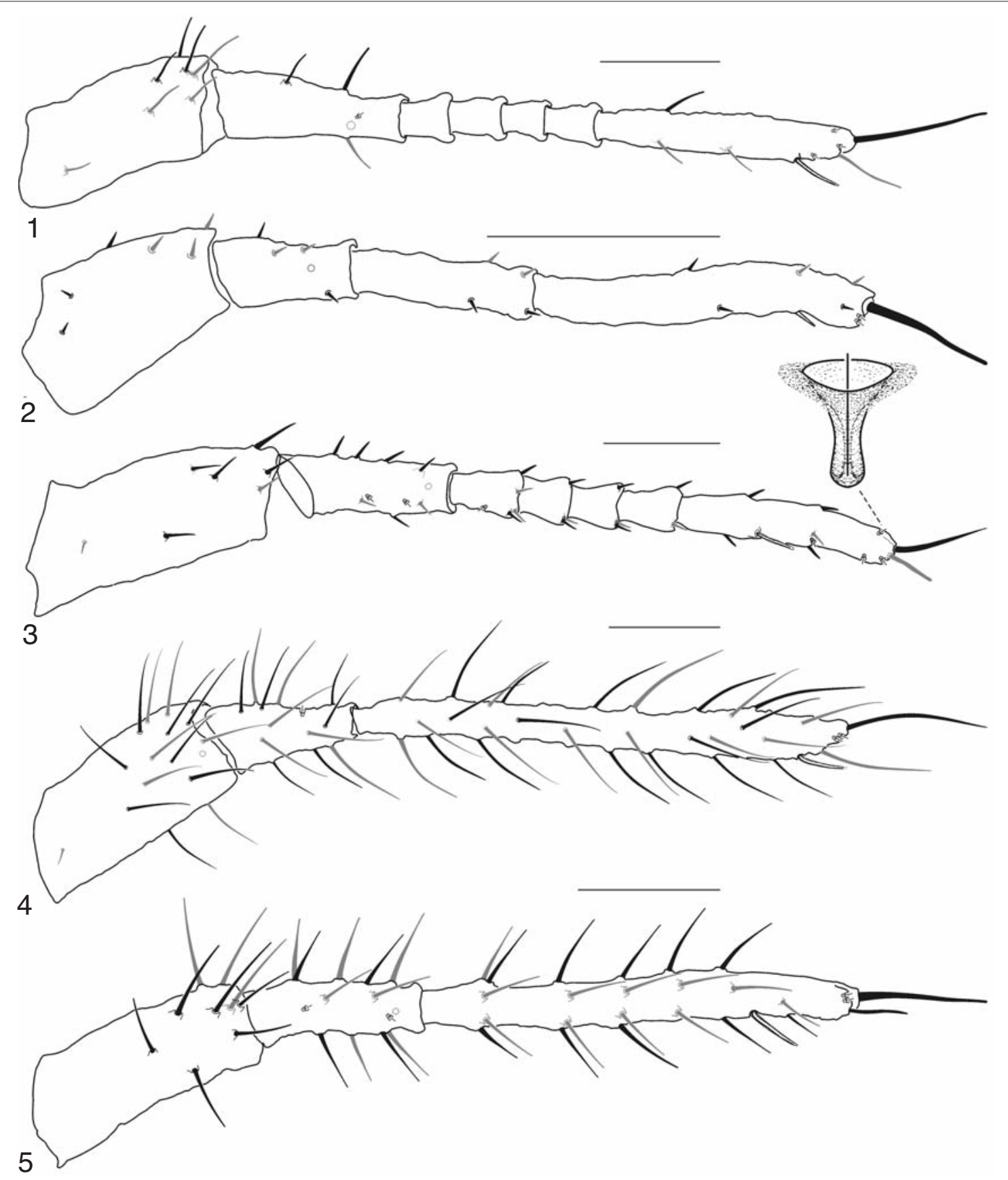

Fig. III - Newsteadia type coeloconic sensilla (Newsteadiinae). 1 - Newsteadia africana Miller \& Kozár, 2 - Newsteadia caledoniensis Kozár \& Konczné Benedicty, 3 - Newsteadia floccosa (De Geer), 4 - Newsteadia monikae Kozár \& Konczné Benedicty, e-Newsteadia morrisoni Kozár \& Konczné Benedicty. Black setae on ventral side, grey setae on dorsal side. Bar $=100 \mu \mathrm{m}$.

\section{Newsteadia monikae Kozár \& Konczné Benedicty} (Fig. III, 4)

Material examined - 1 + Holotype, 3 + Paratypes: New Caledonia, Nouméa, Mt. Koghi, 11.vii.1987, leg. J. Balogh [B.36 HNHM].

Description - Antennae 3 segmented, length of antennal segments: I 192-226, II 128-134, and apical 463-606 $\mu \mathrm{m}$ long; with long hair-like setae present on all segments, 12 on segment I and II, more than 20 on apical segment, longest seta 80-92 $\mu \mathrm{m}$ long; apical seta 96-108 $\mu \mathrm{m}$ long, subapical seta 82-90 $\mu \mathrm{m}$ long; sensory fleshy seta present on apical segment, each 37-50 $\mu \mathrm{m}$ long; with 1
Newsteadia-type cs on segment II, 2 on apical segment. Bs absent.

Newsteadia morrisoni Kozár \& Konczné Benedicty (Fig. III, 5)

Material examined. -1 , , Holotype, Costa Rica, Tarbaca, 1550, 14.i.1993, leg. J. Balogh [B98]; 8 q, Paratypes with the same data as Holotype [B98, B95, B96]; 7 o 9 , Costa Rica, Sierra de la Muerte, 1800, 24.i.1993, leg. J. Balogh [B54, B55].

Description - Antennae 3 segmented; length of antennal segments: I 173-186, II 128-150, and apical 314-350 $\mu \mathrm{m}$ 
long; with long hair-like setae on all segments; with 10 setae on segment I and II and 19 setae on apical segment, longest seta 50-65 $\mu \mathrm{m}$ long; apical seta 99-106 $\mu \mathrm{m}$ long; subapical seta 43-49 $\mu \mathrm{m}$ long; sensory fleshy setae present on apical segment, each 41-51 $\mu \mathrm{m}$ long; 2 Newsteadia-type $c s$ on II and 2 on apical segment. $B s$ absent.

\section{NIPPONORTHEZIINAE}

Description. Antennae 3 or 4 segmented. Setae on antennae spine-like or hair-like. Apical and subapical setae long and hair-like. With 1-4 sensory fleshy setae present on apical segment, sometimes also on subapical segment. Bs relatively thick, conical seta with a pointed or slightly clubbed apex (Figure I, 4), present ventrally, with 2 on apical segment and 1 or 2 on antennal segment II, occasionally also on segment I. Cs absent.

\section{MIXORTHEZIINI}

Description - Antennae 4-segmented. 2 bs present on apical segment, with a slightly capitate apex. 1 sensory fleshy seta present on apical segment and sometimes also on subapical segment. Cs absent.

\section{Jermycoccus boliviensis Kozár \& Konczné Benedicty} (Fig. IV, 1)

Material examined. 1 +, Holotype, Bolivia, La Paz-P. Lineares, 14-16.xi.1971, leg. J. Balogh [B.13 HNHM].

Description. Antennae 4 segmented; length of antennal segments: I $51 \mu \mathrm{m}$, II $45 \mu \mathrm{m}$, III 38, and apical $94 \mu \mathrm{m}$ long; with 4-8 thick seta on each segment; apical seta $90 \mu \mathrm{m}$ long; subapical seta $43 \mu \mathrm{m}$ long; with 1 sensory fleshy seta present on apical segment, $35 \mu \mathrm{m}$ long; 2 bs present on apical segment each with a capitate apex. $C s$ absent.

\section{Mixorthezia morrisoni Konczné Benedicty \& Kozár} (Fig. IV, 2)

Material examined - 1 + Holotype: Cuba, Sierra de la Gran Piedo, 27.v.1979, leg. Pócs [D.Am.145 HNHM].

Description - Antennae 4 segmented; length of antennal segments: I 96, II 64, III 160, and apical $128 \mu \mathrm{m}$ long, setae of antenna spine-like, longest setae $20 \mu \mathrm{m}$ long; apical seta $192 \mu \mathrm{m}$ long; subapical seta $112 \mu \mathrm{m}$ long; with 1 sensory fleshy seta on apical segment, $29 \mu \mathrm{m}$ long, plus a shorter seta on subapical segment; also with $2 b s$ with capitate apex and 2 hair-like sensory seta present on apical segment. $C s$ absent.

\section{Neomixorthezia braziliana Konczné Benedicty \& Kozár} (Fig. IV, 3)

Material examined - 1 \& Holotype, 1 + Paratype: Brasil, 1995, leg. J. Balogh [B.21 HNHM].

Description. Antennae 4 segmented; length of antennal segments: I 103-107, II 56-65, III 168-175, and apical 155$170 \mu \mathrm{m}$ long; setae of antenna hair-like, each 23-30 $\mu \mathrm{m}$ long setae present on apical and subapical segments, and spines each $10 \mu \mathrm{m}$ long on the first 2 segments; apical seta 156-168 $\mu \mathrm{m}$ long; subapical seta 103-132 $\mu \mathrm{m}$ long; with 1 sensory fleshy seta on apical segment, 32-36 $\mu \mathrm{m}$ long, 2 shorter setae present on subapical segment; with $2 b s$ with a capitate apex on apical segment. $C s$ absent.

\section{NIPPONORTHEZIINI}

Description - Antennae 3 or 4 segmented. Two $b s$ with pointed apex present on apical segment and 0-2 on segment II, occasionally on basal segment. 1-4 sensory fleshy seta present on apical segment.

\section{Neonipponorthezia regina Konczné Benedicty} (Figure IV, 4)

Material examined - 1 \& Holotype, 1 \& Paratype, New Guinea, Wau, Nami Creek valley, ex. mosses, 1519.viii.1968, leg. J. Balogh [B.13. HNHM].

Description - Antennae 3 segmented; length of antennal segments: I 128-144, II 176-185, and apical 336-360 $\mu \mathrm{m}$ long; setae on antennae strong, spine-like, each 26-30 $\mu \mathrm{m}$ long; apical seta $166 \mu \mathrm{m}$ long; subapical seta $77 \mu \mathrm{m}$ long; with 4 sensory fleshy seta present on apical segment, each 32-64 $\mu \mathrm{m}$ long; $2 \mathrm{bs}$ with a pointed tip present on apical segment, absent from pedicel; 2 hair-like sensory seta on apical segment. Cs absent.

\section{Nipponorthezia koreana Konczné Benedicty \& Kozár (Fig. IV, 5)}

Material examnined - 1 Holotype, $3 \circ 9$ Paratypes, Korea, Pyongyang city, Mt. Daesong-san, 11.ix.1979, leg. T. Vásárhelyi [As 437 HNHM].

Description - Antennae 3 segmented; length of antennal segments: I 82-92, II 102-122, and apical 250-275 $\mu \mathrm{m}$ long; setae of antenna strong and spine-like, each $12 \mu \mathrm{m}$ long; apical seta 158-168 $\mu \mathrm{m}$ long, subapical seta absent; with 2 sensory fleshy seta on apical segment, each $26-41 \mu \mathrm{m}$ long; $2 b s$ present on apical segment and on segment II and 1 on basal segment; 2 hair-like sensory setae each with slightly capitate apex on apical segment. Cs absent.

\section{Nipponorthezinella guadalcanalia (Morrison)} (Fig. IV, 6)

Material examined - $3 \circ \circ$, New Caledonia, Ile de Pius, leaf litter, 18-24.v.1987, leg. J. Balogh [B. 4-5 HNHM]; 2 오, New Caledonia, Farino, soil, 19.vii-25.viii.1982, leg. J. Balogh [82.B.2. HNHM]; 2 qo, New Caledonia, Maré, on roots of epiphytons, 26.v-9.vi.1978, leg. J. Balogh [B.19. HNHM]; 2 Q 9 , Thailand, 1994, leg. S. Mahunka [As 712 HNHM]; 3 우, Seychelles, Cascade East, 10.xii.1975, leg. Fjellberg [431 PPI].

Description - Antennae 3 segmented; length of antennal segments: I 72-82, II 108-144, and apical 227-298 $\mu \mathrm{m}$ long; setae strongly curved and hair-like, each 25-30 $\mu \mathrm{m}$ long with a long cylindrical basal collar; apical seta 130-150 $\mu \mathrm{m}$ long; subapical setae 35-41 $\mu \mathrm{m}$ long; with 1 (rarely 2) sensory fleshy seta on apical segment, each $40-45 \mu \mathrm{m}$ long; $2 b s$ present on apical segment and 3 on pedicel; 2 hair-like sensory seta present on apical segment. $C s$ absent.

\section{Orthezinella hispanica Silvestri (Fig. IV, 7)}

Material examined - 3 우: Spain, Algeciras, 03.i.1923, leg. F. Silvestri.

Description - Antennae 4 segmented; length of antennal segments: I 82-103, II 113-122, III 154-187 $\mu \mathrm{m}$ long, and apical 133-150 $\mu \mathrm{m}$ long; setae spine-like, each $20 \mu \mathrm{m}$ long; apical seta 134-140 $\mu \mathrm{m}$ long; subapical seta 38-41 $\mu \mathrm{m}$ long; with 1 sensory fleshy seta on apical segment and 2 on subapical segment, each 31-48 $\mu \mathrm{m}$ long; $2 \mathrm{bs}$ present on apical segment and 2 on segment II; 2 hair-like sensory seta on apical segment. $C s$ absent.

\section{ORTHEZIOLINI}

Description - Antennae 3 or 4 segmented. Pseudo-basal (first) segment fused with eye. Setae on antennae spine-like or hair-like. Apical and subapical setae long and hair-like. One sensory fleshy seta present on apical segment, occasionally also on basal segment. $B S$ present on ventral 

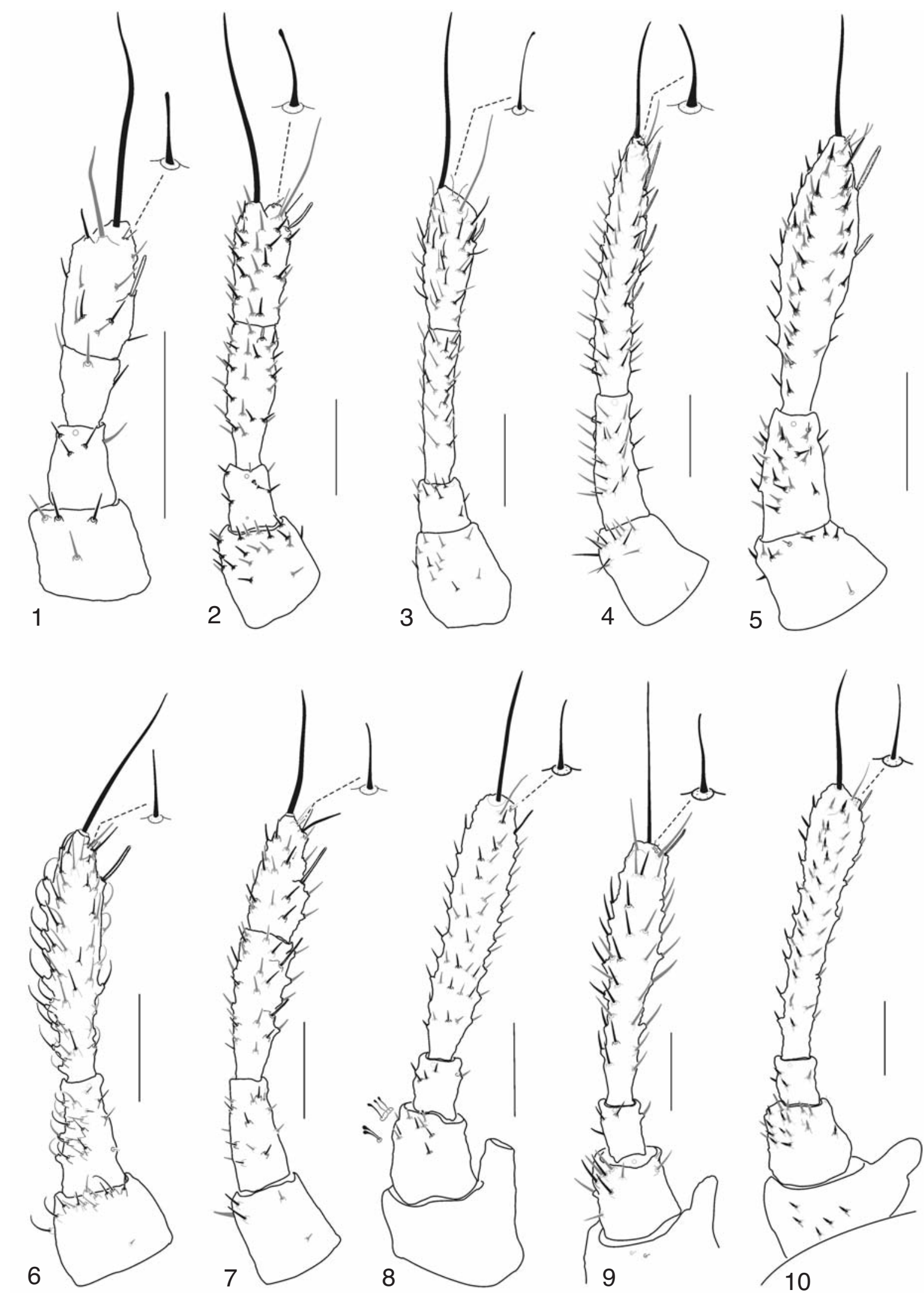

Fig. IV - Basiconic sensilla (Nipponortheziinae).a-Jermycoccus boliviensis Kozár \& Konczné Benedicty, b-Mixorthezia morrisoni Konczné Benedicty \& Kozár, c-Neomixorthezia brazilana Konczné Benedicty \& Kozár, d-Neonipponorthezia regina Konczné Benedicty, e-Nipponorthezia koreana Konczné Benedicty \& Kozár, f-Nipponorthezinella guadalcanalia (Morrison, 1952), g-Orthezinella hispanica Silvestri, h-Ortheziola vejdovkyi Šulc, i-Ortheziolacoccus matileferreroae Kozár \& Miller, j-Ortheziolamameti guinensis (Morrison). Black setae on ventral side, grey setae on dorsal side. Bar $=100 \mu \mathrm{m}$. 
side of antennae as relatively thick, conical setae with a pointed apex, with 2 on apical segment and on 1 or 2 on segment II and 0 or 1 on basal segment. Cs absent.

Ortheziola vejdovkyi Šulc (Fig. IV, 6)

Material examined - 1 , Austria, Vienna, soil with mosses, 26.vii.2013, leg. K. Fetykó [11132 PPI]; 1 q, Hungary, Nagykovácsi, Júlia-Major, rocky grass land, D-vac, 12.x.1999, leg. É. Szita \& E. Botos [6061 PPI]; 1 q, Hungary, Bátorliget, ősláp, D-vac, 7.xi.2008, leg. F. Samu [8521 PPI]; 1 q, Hungary, Pátka, Aszal-Völgy, D-Vac, 24.ix.2012, leg. É. Szita \& E. Botos [10660 PPI]; 1 ㅇ, Hungary, Aggteleki NP, Jósvafö, Haragistya, mosses, 15.vii.2009, leg.Kozár [8816 PPI]; 1 q, Armenia, Tsakhkadzor, 1800-2300, 1.x.1982, leg. O. Merkl [As 569 HNHM]; 2 오, Slovenia, Bohinjska Bela, leaf litter, 22.viii.2009, leg. J. Kontschán [10925 PPI]; 1 †: Romania, Békás-szoros, 20.vii.1983, leg. K. Sim [10905 PPI]; 1 ㅇ, Romania, Mehadia, leaflitter, 25.x-5.xi.2007, leg. J. Kontschán [10825 PPI]; 2 우, Romania, Tordaihasadék, 28.ix.2001, leg. T. Szüts [10844 PPI]; 1 ๆ, Switzerland, Valais, s/Venayaz, 700, 7.x.1980, leg. C. Besuchet [8864 PPI].

Description - Antennae 4 segmented; length of antennal segments: I 84-113, II 62-84, III 309-346 $\mu \mathrm{m}$ long; all segments of antennae covered with a moderate number of spine-like setae, each straight and apically acute; apical segment with 35-45 setae, longest seta 12-16 $\mu \mathrm{m}$ long; apical seta 154-163 $\mu \mathrm{m}$ long; subapical seta $51-59 \mu \mathrm{m}$ long; with 1 sensory fleshy seta on apical segment, each 30-48 $\mu \mathrm{m}$ long; 2 bs present on apical segment and 1 on pedicel; a group of 2 or 3 blunted sensory setae present on ventral side of basal segment. Cs absent.

\section{Ortheziolacoccus matileferreroae Kozár \& Miller} (Fig. IV, 9)

Material examined - $1 q$ Holotype, 4 qq Paratypes, Tanzania, Poroto, ex. Arundinaria alpina, 22.i.1972, leg. Pócs [160 PPI].

Description - Antennae 4 segmented; length of antennal segments: I 99-131, II 58-96 $\mu \mathrm{m}$, III 347-390 $\mu \mathrm{m}$ long; all segments of antennae covered with small number of robust, straight, apically acute setae; apical segment with 32-40 setae, longest setae eac34-44 $\mu \mathrm{m}$ long; apical seta 212-232 $\mu \mathrm{m}$ long; subapical seta 67-74 $\mu \mathrm{m}$ long; sensory fleshy seta present on apical segment, each 32-38 $\mu \mathrm{m}$ long; $2 \mathrm{bs}$ present on apical segment and 1 on basal segment; 2 hairlike sensory seta present on apical segment. $C s$ absent.

\section{Ortheziolamameti guinensis (Morrison) (Fig. IV, 10}

Material examined - 1 : : Ghana, Nyankapala, 5.viii.1968, leg. Y.S. Endrödy.

Description - Antennae 4 segmented; length of antennal segments: I 110-124, II 61-82, III 398-422 $\mu \mathrm{m}$ long; all segments of antennae covered with moderate number of robust, slightly lanceolate setae, apical segment with 48-52 setae, longest setae each 14-25 $\mu \mathrm{m}$ long; apical seta $153 \mu \mathrm{m}$ long; subapical seta 49-54 $\mu \mathrm{m}$ long; with 1 sensory fleshy seta on apical segment and 1 on basal segment, each $31 \mu \mathrm{m}$ long; 2 bs present on apical segment and 2 on basal segment. Cs absent.

\section{PHYLOGENETIC RELATIONSHIP}

By adding the antennal characters to the morphological matrix of VeA \& GRimaldi (2012), the MP analysis retrieved 461 most parsimonious trees of 296 steps (Fig. I). Modifying the above morphological matrix did not result in a significant change of topology with almost all groupings remaining the same as in VEA \& GRIMALDI (2012). The only difference is a loss of resolution within the clade including the Ortheziolini and Mixortheziini.

Three main lineages are distinguished by their type sensilla (Fig. V): (1) the Ortheziinae (including both Arctortheziini and Ortheziini) all possess $c s$, which according to character optimization originated in this group; (2) the Newsteadiinae, which includes only the genus Newsteadia, is characterized by a different type of $c s$, the Newsteadia-type $c s$, and (3) the last group includes members of the Mixortheziini, Nipponortheziini and Ortheziolini, which all possess only $b s$.

When optimizing the type of sensillum (character 70) on the strict consensus (Fig. V) using parsimony, bs are inferred as the ancestral type of sensillum at least within the Ortheziidae. Therefore, despite their similarity, the two types of $c s$ have a different evolutionary origin, one originating in the Ortheziinae and the other in the Newsteadiinae.

When mapping the habitat for each terminal, a trend linking the type of sensilla and evolution of habitat seems to appear. First, we have groups feeding on aerial parts and possessing $c s$, as in Ortheziinae. However, this excludes the tribe Arctortheziini, mainly found either in the leaf litter or feeding on plant roots. Second, the majority of species within Newsteadia are ground-dwelling, with some exceptions where species are found in the aerial parts of plants (e.g., N. americana Morrison, N. floccosa). Overall, the Newsteadia-type $c s$ is linked to a grounddwelling habitat. Third, the majority of the Ortheziidae which have $b s$ either feed on plant roots or are grounddwelling.

\section{DISCUSSION}

LARINK (1986) and WALTHER (1983) suggested that the number and distribution of sensilla on the antennal segments are probably of taxonomic and phylogenetic significance in insects. Here, we found that the type of antennal sensillum within the Ortheziidae is indeed taxonomically informative. The family can be divided into three main lineages based on their type of sensillum: (i) coeloconic sensilla, (ii) Newsteadia-type coeloconic sensilla, and (iii) basiconic sensilla. These three lineages are the same as those recovered in the phylogenetic analysis of VEA \& GRimaldi (2012). Based on the character optimization in our phylogenetic hypothesis, $b s$ seem to be the ancestral state to the two types of $c s$. Although Newsteadia-type $c s$ and regular $c s$, have some similarities, these are herein considered to be morphologically different. Newsteadia-type cs have thicker walls and longer setae than those on "typical" cs. It is thought that these differences led to of different lineages in ortheziid evolution.

Koteja (1980) also described $b s$ and $c s$ of different families within the Coccoidea and did mention their phylogenetic importance. He put forward two conclusions: (i) the $b s$ are probably the primary type, because they occur in Phenacoleachia, and partly in the Monophlebidae and Coelostomidiidae, (ii) the $c s$ underwent a secondary transformation towards the $b s$ in some specialized groups such as part of Ortheziidae, and in some neococcoid lineages. Although our study partly supports Koteja evolutionary views, we believe that this separation occurred not only because of the age of the families but also because of the habitat where the insects live. In this study, we 


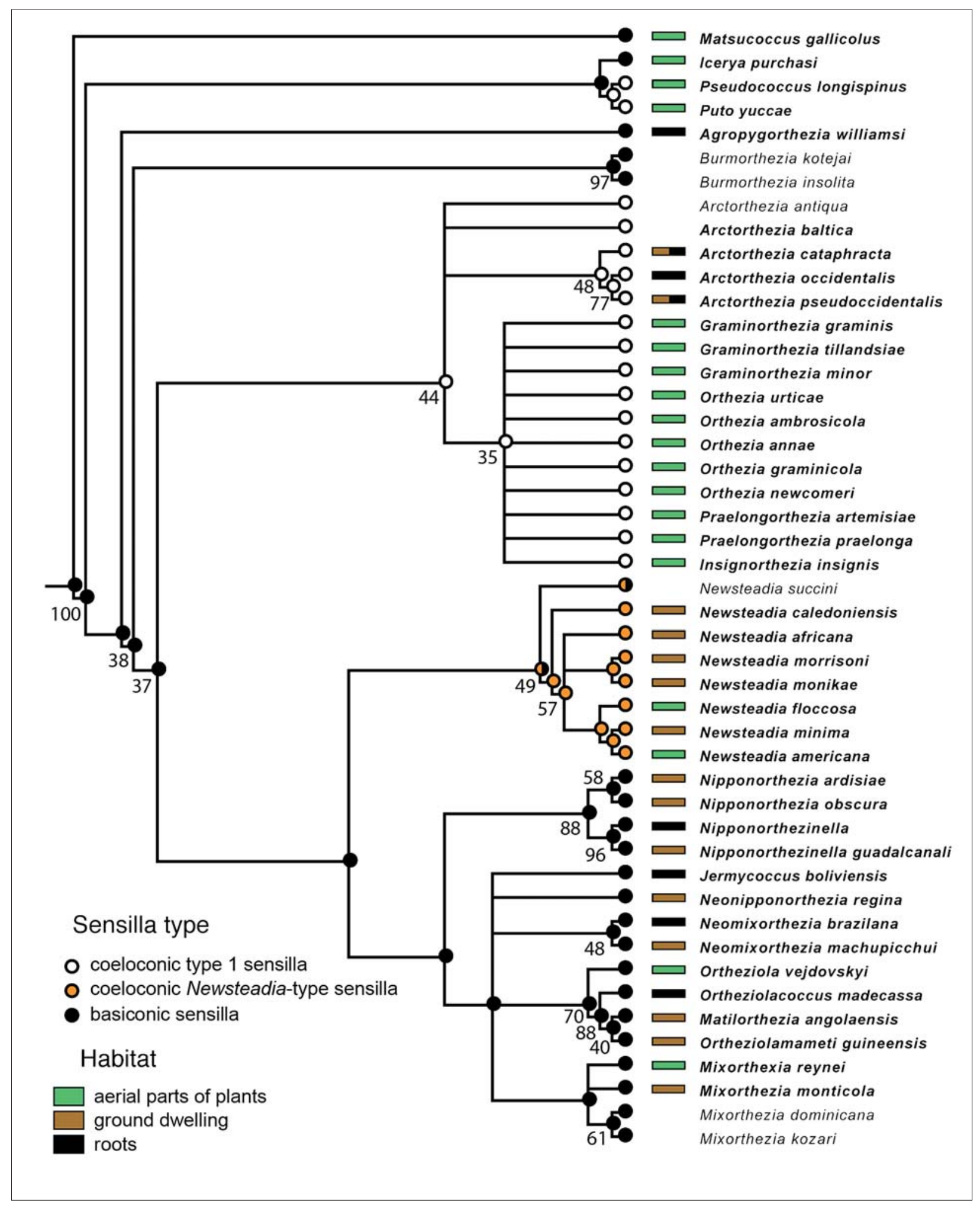

Fig. V - Strict consensus of the 461 most parsimonious trees obtained from a Maximum Parsimony analysis with TNT. L= 296; CI $=0.35$, $\mathrm{RI}=0.74$. Ancestral reconstruction of the type of antennal sensilla (Character 70) was performed with Mesquite using unordered parsimony and is shown on the tree as coloured circles. Habitat is indicated with the coloured rectangles. Bootstrap values $>20 \%$ are indicated at each node.

showed that $b s$ occurs in insects (such as Ortheziolini and Nipponortheziini) that live on mosses, leaf litter and in the soil with a humid ecosystem. On the other hand, all insects that live on aerial plant parts in this study have $\operatorname{cs}$ (i.e., Ortheziinae and Newsteadiinae).

KoteJA (1980) also indicated that the $b s$ and $c s$ represent organs of the same origin and function, being present in similar number and situated on the same segments. In this study, it is shown that the location and number of the sensilla can be variable in different species, but the antennal apical segments always bear sensilla. To better understand their role, further studies are necessary to determine their number and location not only in the Ortheziidae but also in other families of Coccoidea. 


\section{ACKNOWLEDGEMENTS}

The first author (MBK) is grateful to TUBITAK financial support for the study of the Ortheziidae in Hungary. The authors wish to thank to Zsuzsanna Konczné Benedicty (PPI, Budapest, Hungary) for supporting our laboratory work. Moreover, we thank Dr. Takumasa Kondo (Corpoica, Palmira, Colombia) for comments on the manuscript.

\section{REFERENCES}

Goloboff P.A., Farris J.S., Nixon K.C., 2008 CLADISTICS TNT, a free program for phylogenetic analysis. - Cladistics, 24: 774-786 (DOI: 10.1111/j.10960031.2008.00217.x).

KEIL T.A., 1999 - Morphology and development of the peripheral olfactory organs. In: Hansson, B.S. (Ed.), Insect olfaction. Springer-Verlag, Berlin Heidelberg, pp. 5-47 (DOI: 10.1007/978-3-662-07911-9-2).

KотеJA J., 1985 - Essay on the prehistory of the scale insects (Homoptera, Coccinea). - Ann. Zool. (Warsaw), 38: 461-504.

Koteja J., 1986 - Morphology and taxonomy of male
Ortheziidae (Homoptera, Coccinea). - Pol. Pismo Entomol., 56: 323-374.

KozÁr F., 2004 - Ortheziidae of the World. Plant Protection Institute, Hungarian Academy of Sciences, Budapest.

LARINK O., 1986 - Pattern of antennal sensilla in some Lepismatidae (Insecta: Zygentoma); 2nd International Seminar on Apterygota, pp. 237-242.

Maddison W.P., Maddison D.R., 2014 - Mesquite: A modular system for evolutionary analysis. Version 3.01. Accessed 28 November 2014. Online at http:// mesquiteproject.org

O'LeARY M.A., KAUfMAN S.G., 2012 - MorphoBank 3.0: Web application for morphological phylogenetics and taxonomy. http://www.morphobank.org.

SCHNEIDER D., 1964 - Insect antennae. - Ann. Rev. Entomol., 9: 103-122.

VEA I., GRIMALDI D.A., 2012 - Phylogeny of ensign scale insects (Hemiptera: Coccoidea: Ortheziidae) based on the morphology of recent and fossil females. - Syst. Entomol., 37: 758-786 (DOI: 10.1111/j.1365-3113.2012.00638.x).

WALTHER J.R., 1983 - Antennal patterns of sensilla of the Hymenoptera. A complex character of phylogenetic reconstruction. - Verh. natwiss. Ver Hamburg N.F., 26: 373-392. 